www.jmscr.igmpublication.org

Impact Factor (SJIF): 6.379

Index Copernicus Value: 71.58

ISSN (e)-2347-176x ISSN (p) 2455-0450

crossref DOI: https://dx.doi.org/10.18535/jmscr/v6i6.135

Journal Of Medical Science And Clinical Research

IGM Publication

An Official Publication of IGM Publication

\title{
Circulating Cathodic Antigen Rapid Test in the Diagnosis of Urinary Schistosomiasis among Rice Farmers in Parts of Kaduna State, Nigeria
}

\author{
Authors \\ Awua, Yuana*1, Inabo, Helen Ileigo $^{1}$, Yakubu, Sabo Ezekiel ${ }^{1}$ \\ Okubanjo, Oluyinka Oluseyi ${ }^{2}$ \\ ${ }^{1}$ Department of Microbiology, Faculty of Life Sciences, Ahmadu Bello University, Zaria \\ ${ }^{2}$ Department of Protozoology and Entomology, Faculty of Veterinary Medicine, Ahmadu Bello University, \\ Zaria \\ *Corresponding Author \\ Awua, Yuana \\ Department of Microbiology, Faculty of Life Sciences, Ahmadu Bello University, Zaria \\ Email: yuaawua@gmail.com., Mobile Number: +234 8065694225
}

\begin{abstract}
Background: Schistosomiasis is the most prevalent among water-based parasitic infections which is caused by infection from the genus Schistosoma. Urinary schistosomiasis is a human disease which is caused by Schistosoma haematobium.

Methods: A cross-sectional study was carried out among randomly selected 600 rice farmers, comprising of 354 males and 246 females from Kaduna State, Nigeria. The point-of-care (POC) circulating cathodic antigen (CCA) urine test strip (Rapid Medical Diagnostics) was evaluated for the diagnosis of active Schistosoma haematobium infection while urine filtration technique using polycarbonate membrane filter (Sterlitech, Kent, WA, USA) was employed for microscopic processing of urine samples. Chi square $\left(\chi^{2}\right)$ test was used to determine the association between variables and infection at $95 \%$ confidence interval at $p$ $=0.05$.
\end{abstract}

Results: A prevalence of $15.5 \%$ was recorded among rice farmers and had a statistically significant association with the detection of $S$. haematobium antigen $(p<0.05)$ in relation to the different locations studied. Microscopy revealed a prevalence of $0.83 \%$. Age was significantly associated with urinary schistosomiasis $(p<0.05)$ using the CCA test strip, with those in age group 15-24 years having the highest prevalence of $27.5 \%$. The sensitivity of CCA was $85 \%$ while the specificity was $93 \%$. Marital status of rice farmers was significantly associated with $S$. haematobium antigen in urine $(p<0.05)$ with singles having the highest prevalence of $20.0 \%$.

Conclusion: POC-CCA urine test in combination with microscopy can be helpful in rapidly detecting active infection in urine of rice farmers who are shedding or not shedding Schistosoma haematobium ova.

Keywords: Circulating cathodic antigen (CCA), Schistosoma haematobium, rice farmers, rapid test.

\section{Introduction}

Schistosomiasis is considered one of the Neglected Tropical Diseases (NTDs) and is estimated to affect 249 million people worldwide, of which 224 million affected people live in sub Saharan Africa ${ }^{(1)}$ with 732 million people at risk 
of infection in known transmission areas ${ }^{(2)}$. It is a prevalent tropical disease ranking second to malaria and killing an estimated 280,000 people each year in the African region alone ${ }^{(3)}$. In subSaharan Africa, Nigeria carries the heaviest burden with an estimated 29 million cases of infection $^{(4,5)}$.

Urinary schistosomiasis is a human disease caused by the trematode Schistosoma haematobium. It is endemic in 78 countries affecting over 200 million people $^{(1)}$ and continues to be a global public health concern in the developing world. In Nigeria, urinary schistosomiasis has a wide distribution and the low resource communities are mostly plagued by the disease due to the fact that it is known to affect the working capacity of rural inhabitants owing to the weakness and lethargy it induces $^{(6)}$. Though the majority of people in endemic areas have only light infections or no symptoms, the impact of urinary schistosomiasis on economic condition and the general health situation is $\operatorname{high}^{(7)}$.

Urine microscopy for detection of S. haematobium ova is considered one of the most conclusive diagnostic criteria or "gold standard" for urinary schistosomiasis $^{(8)}$. Unfortunately, it is cumbersome, time-consuming and too technical. This limitation therefore dictates the need for a simple, fast, sensitive, field-applicable and reliable diagnostic method for the detection of infected persons and for consistent screening in ensuring the proper management of the disease especially among rice farmers in low resource communities of Nigeria.

More recently, circulating cathodic antigen (CCA) urine test strips for rapid schistosomiasis diagnosis in urine have been developed ${ }^{(9)}$. Studies carried out to determine the field applicability has so far demonstrated that the method is valuable for detection in endemic communities ${ }^{(10)}$.

\section{Materials and Methods}

\section{Study Area}

This study was conducted in randomly selected parts of Kaduna State; Giwa, Igabi, Kajuru and
Kachia local government areas respectively. Kaduna State lies between latitudes $10^{\circ} 21^{\prime}$ and $10^{\circ} 33^{\prime}$ North of the equator and longitudes $7^{\circ} 45^{\prime}$ and $7^{\circ} 75^{\prime}$ East of the Greenwich meridian and has 23 local government areas. The State has both wet and dry seasons with the wet season commencing in the month of April in southern part of the state and between May and June in the northern part. Rainfall is heaviest in the southern part of the state and decreases northwards with mean annual rainfall varying between $942 \mathrm{~mm}$ and $1000 \mathrm{~mm}$ lasting from May to October. The dry season sets in immediately and is characterized by harmattan (that blows between the end of November and the Middle of March) with temperature range of $18^{\circ} \mathrm{C}$ to $26^{\circ} \mathrm{C}$ and heat period with a temperature that ranges from $32^{\circ} \mathrm{C}$ to $39^{\circ} \mathrm{C}$.

\section{Study Design}

This study was a cross-sectional study and a probability multistage sampling technique was employed.

\section{Collection of Urine Samples}

A total of 600 rice farmers were provided with a clean, wide-mouthed screw cap universal bottle carrying the same identification number with the one on the questionnaire. The urine samples were collected between 10 am and $2 \mathrm{pm}$. Ten (10) $\mathrm{ml}$ of urine was filtered on the field using a plastic syringe with a filter-holder containing a $13 \mathrm{~mm}$ diameter polycarbonate filter and $12 \mu \mathrm{m}$ pore size (Sterlitech, Kent, WA, USA). The remaining 10 $\mathrm{ml}$ was tested for circulating schistosome antigen using the circulating cathodic antigen (CCA) urine cassette test strips.

\section{Parasitological Examination}

All the filtered urine on polycarbonate membrane filters (Sterlitech, Kent, WA, USA) from the field were examined using x 10 and $x 40$ objective lens of a light microscope for the eggs of Schistosoma haematobium and were confirmed using an Atlas of Parasitology It was reported as number of eggs/ $10 \mathrm{ml}$ of urine. 


\section{Detection of Circulating Schistosome Antigen (CSA)}

This test was carried out using a circulating cathodic antigen (CCA) cassette test strip (Rapid Medical Diagnostics). A drop of the urine was placed into the circular well of the test cassette using a pipette bulb and allowed to absorb entirely into the specimen pad. One (1) drop of buffer was added to the test cassette containing the urine sample. The results were read at exactly 20 minutes.

\section{Results}

Out of the 600 urine samples of rice farmers examined by microscopic analysis, a prevalence of $0.83 \%$ (5/600) rice farmers had Schistosoma haematobium ova in their urine while $15.5 \%$ (93/600) of them had the Schistosome circulating antigen as detected by the CCA cassette test strip (Rapid Medical Diagnostics, South Africa) as shown in Figure 1.

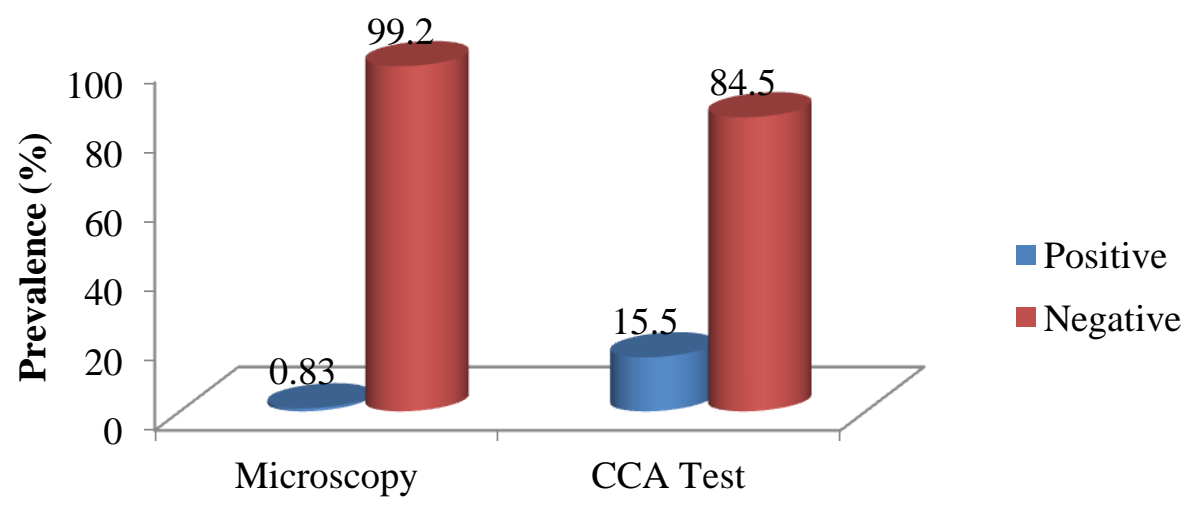

Test Methods

Figure 1: Prevalence of urinary schistosomiasis among rice farmers by CCA and microscopy

The results obtained from the locations showed a statistical significant association with urinary schistosomiasis using the CCA test strip for antigen detection $\left(\chi^{2}=38.291, \mathrm{p}=0.000^{*}\right)$. Results from microscopic detection of ova of $S$. haematobium did not show any significant association with urinary schistosomiasis $\left(\chi^{2}=\right.$ $3.817, \mathrm{p}=0.282$ ) for the different study locations. (Table 1).

Table 1: Prevalence of urinary schistosomiasis among rice farmers by sampling locations in parts of Kaduna State, Nigeria

\begin{tabular}{lccc}
\hline $\begin{array}{l}\text { Location } \\
\text { (LGA) }\end{array}$ & $\begin{array}{c}\text { Number of } \\
\text { samples } \\
\text { examined (N=600) }\end{array}$ & $\begin{array}{c}\text { Microscopy } \\
\text { Positive (\%) }\end{array}$ & $\begin{array}{c}\text { CCA }^{\text {\#̈f }} \\
\text { Positive (\%) }\end{array}$ \\
\hline Giwa & 150 & $3(2.0)$ & $17(11.3)$ \\
Igabi & 150 & $0(0.0)$ & $5(3.4)$ \\
Kajuru & 150 & $1(0.7)$ & $42(28.0)$ \\
Kachia & 150 & $1(0.7)$ & $29(19.3)$ \\
\hline
\end{tabular}

$\#: \chi^{2}=3.817, \mathrm{p}=0.282$

$\# \#: \chi^{2}=38.291, \mathrm{p}=0.000$

Key: LGA - Local Government Area

CCA - Circulating cathodic antigen

In relation to sex, there was however no statistical significant difference between the male and female rice farmers $(p>0.05)$ as shown in Table 2 . 
The prevalence of urinary schistosomiasis by antigen detection among rice farmers in relation to age revealed a statistical significant difference using the Chi square $\left(\chi^{2}\right)$ test analysis $\left(\chi^{2}=6.789\right.$, $\mathrm{p}=0.020)$ between the age group. There was however no statistical significant difference $\left(\chi^{2}=\right.$ 1.034, $\mathrm{p}=0.341)$ between age groups by microscopic analysis (Table 2).

Table 2: Age-related prevalence of urinary schistosomiasis among rice farmers in parts of Kaduna State, Nigeria

\begin{tabular}{lccc}
\hline Factor & $\begin{array}{c}\text { Number } \\
\text { examined (N= 600) }\end{array}$ & $\begin{array}{c}\text { Microscopy } \\
\text { positive (\%) }\end{array}$ & $\begin{array}{c}\text { CCA \#\# } \\
\text { positive (\%) }\end{array}$ \\
\hline Sex $*$ & & & \\
Male & 354 & $3(0.85)$ & $53(14.9)$ \\
Female & 246 & $2(0.81)$ & $40(16.3)$ \\
Age group** & & & \\
(Years) & & & \\
$5-14$ & 86 & $1(1.2)$ & $11(12.8)$ \\
$15-24$ & 171 & $1(0.6)$ & $16(27.5)$ \\
$25-34$ & 79 & $1(1.3)$ & $7(6.7)$ \\
$35-44$ & 105 & $1(1.0)$ & $7(7.3)$ \\
$45-54$ & 96 & $0(0.0)$ & $1(3.0)$ \\
$55-64$ & 33 & $1(3.0)$ & $4(13.3)$ \\
$65-74$ & 30 & $0(0.0)$ & \\
\hline
\end{tabular}

$\#: * \chi^{2}=0.02, p=0.967 ;$ OR $=0.963(1.161-5.586)$

$* * \chi^{2}=1.034, \mathrm{p}=0.341$

\#\#: * $\chi^{2}=0.203, p=0.653 ; O R=0.902(0.577-1.414)$

$* * \chi^{2}=6.789, \mathrm{p}=0.020$

Key: CCA - Circulating cathodic antigen

Marital status was found to be significantly associated with urinary schistosomiasis among rice farmers using the CCA test strip $\left(\chi^{2}=10.387\right.$,

Table 3: Prevalence of urinary schistosomiasis in association with marital status of the rice farmers in parts of Kaduna State, Nigeria

\begin{tabular}{lccc} 
Risk factor & $\begin{array}{c}\text { Number } \\
\text { examined }(\mathbf{N = 6 0 0 )}\end{array}$ & $\begin{array}{c}\text { Microscopy" } \\
\text { positive (\%) }\end{array}$ & $\begin{array}{c}\text { CCA "'" } \\
\text { positive (\%) }\end{array}$ \\
\hline Marital status & & & $59(20.0)$ \\
Single & 295 & $2(0.7)$ & $30(11.7)$ \\
Married & 256 & $2(0.8)$ & $3(14.3)$ \\
Divorced & 21 & $1(4.8)$ & $1(3.6)$ \\
Widowed & 28 & $0(0.0)$ & \\
\hline
\end{tabular}

$\#: \chi^{2}=4.524, \mathrm{p}=0.210$

$\# \#: \chi^{2}=10.387, \mathrm{p}=0.016$

Key: CCA - Circulating cathodic antigen

The sensitivities and specificities of CCA in comparison with microscopy is as shown in Table 4.

Table 4: Sensitivity and specificity of POC-CCA against urine filtration test techniques

\begin{tabular}{lcc}
\hline Variable & Sensitivity (\%) & Specificity (\%) \\
\hline CCA & 85.0 & 93.0 \\
Urine filtration & 63.0 & 86.0 \\
(Microscopy) & & \\
\hline
\end{tabular}

Key: CCA- Circulating Cathodic Antigen 


\section{Discussion}

Based on our findings from this study, we observed a prevalence of $0.83 \%$ by urine filtration in microscopic detection of the ova and $15.5 \%$ of circulating schistosome antigen, using the circulating cathodic antigen (CCA) test strip among rice farmers in some parts of Kaduna State, Nigeria.

The low prevalence by microscopy could be due to the fact that only single urine samples from rice farmers were examined and probably this might have led to missing some of the eggs as a result of the circadian rhythm or daily excretion of the eggs by each rice farmer. This of course was a major limitation in this study which probably accounted for the higher prevalence in the active infected cases as detected by the CCA test strip. The CCA which is rapid immunodiagnostic test detected $S$. haematobium antigen in rice farmers who were presumed ova negative by microcopy.

This study was carried out during the rice growing period in flooded areas of the rainy season and the population of the intermediate host snails responsible for habouring the infective larvae could have reduced. The infection rate and distribution of schistosome intermediate host decreases due to the seasonal changes as a result of flooding which may eventually lead to a decrease in the snail density in given foci ${ }^{(11)}$.

A study carried out in a rice-growing community during the rainy season recorded a prevalence of $1.6 \%$ of urinary schistosomiasis among individuals who resided in rice-growing communities of Ogun State, Nigeria ${ }^{(12)}$. This finding however contrasts the prevalence reported among rural Ezza farmers in Ebonyi State, Nigeria ${ }^{(13)}$. The different prevalence could be attributed to variations in geographic and environmental settings as well as socio-cultural beliefs as it has been shown that schistosomiasis is focally distributed and prevalent rates differ between communities and locations of Nigeria ${ }^{(14,15)}$. The presence of impounded water bodies like dams for instance in different locations play a vital role in the transmission of urinary schistosomiasis among rice farmers. The immunodiagnostic test using the CCA test strip detected active cases among rice farmers with $S$. haematobium infection.

It was found in this study that, there was significant circulating schistosome antigen as detected by CCA among rice farmers of the age group 15-24 years as compared to rice farmers in age group 55-64 years. This could be a combined function of immunity and water contact habits of the rice farmers. A similar pattern in the peak prevalence of $S$. haematobium among rural Ezza farmers ${ }^{(13)}$ was reported. Younger persons between 10-19 years contribute bulk of the S. haematobium shed into the environment ${ }^{(16)}$. In the older persons, there is usually a progressively increasing level of naturally acquired immunity against $S$. haematobium infection with changes in water contact habits.

The effect of marital status on the prevalence of urinary schistosomiasis in this study may be due to the fact that singles are more likely to visit streams or other bodies of water ${ }^{(17)}$ besides the flooded rice fields where they may come into contact with cercariae of schistosome.

In comparison with microscopy, the circulating cathodic antigen (CCA) had a sensitivity of $85 \%$ and a specificity of $93 \%$ among rice farmers with active Schistosoma haematobium infection. This assay has proved to be a useful test for the diagnosis of $S$. haemaobium. A sensitivity of $88.2 \%$ and $47.7 \%$ with a specificity of $100 \%$ and $75.8 \%$ respectively has been reported ${ }^{(10,18)}$.

\section{Conclusion}

The CCA test was able to detect 93 positive cases with 5 microscopically positive cases inclusive. This indicates that circulating cathodic antigen (CCA) which can act as specific markers for the presence of $S$. haematobium and can be potentially used to complement microscopy in the screening of active infection.

\section{Recommendation}

We recommend that, circulating cathodic antigen (CCA) can be used as an effective rapid 
diagnostic tool in monitoring urinary schistosomiasis. This will help prevent any debilitating health effect of the disease.

\section{Acknowledgement}

We would like to thank the study participants for their consent to carry out this research.

\section{Declaration}

\section{Ethical consideration}

Approval for the research was obtained from the Research and Ethical Committee (REC) of the Kaduna State Ministry of Health with reference number (MOH/ADM/744/VOL.1).

\section{Consent to publish}

All authors have read the final version of the manuscript and have given approval for it to be published. Informed consent was obtained from rice farmers to share the findings of this research through publication.

\section{Competing interests}

All authors declare that they have no competing interests.

\section{Author's Contribution}

The authors declare no conflict of interest. AY designed the study, collected and analysed data and wrote the manuscript. IHI, YSE and OO revised the manuscript. All authors proof read the final version of the manuscript before submission.

\section{References}

1. World Health Organization (WHO). Schistosomiasis.www.who.int/mediacentre/fa ctsheetsfs 115/en/2016.

2. King $\mathrm{CH}$. Parasites and poverty: the case of schistosomiasis. Acta Tropica, 2010; 113:95 104.

3. Centers for Disease Control and Prevention (CDC). Neglected Tropical Diseases.cdc.gov. 2011.

4. Hotez PJ and Kamath A.Neglected tropical diseases in sub-Saharan Africa: review of their prevalence, distribution and disease burden. PLo S Negl Trop Dis. 2009;3:e412.
5. Adenowo AF, Oyinloye BE, Ogunyinka BI, Kappo AP. Impact of Human schistosomiasis in sub-Saharan Africa. Braz $J$ of Infect Dis. 2015; 19(2):196-205.

6. Uneke CJ, Oyibo PG, Ugwuoru CDC, Nwanokwai AP, Iloegbunam RO Urinary Schistosomiasis among School Age Children in Ebonyi State,Nigeria. Intern JLab Med.2007;2(1):1.

7. Chidozie EU, Duniyan SY. Urinary schistosomiasis epidemiological survey of urinary schistosomiasis among childrenin selected schools: A preliminary study in Minna, Nigeria. AfrJ Biotechnol.2008;7 (16):2773-2776.

8. Aljafari AS, Abosalif KO, Shammat IM, Shiff CJ, Ahmed AA. Evaluation of Polymerase Chain Reaction and Millipore filtration method for the diagnosis of Schistosoma haematobium: A comparative study. EC Microbiol. 2015;1 (3):150-154.

9. Van Dam GJ, Wichers JH, Ferreira TM, Ghati D, van Amerongen A, Deelder AM Diagnosis of schistosomiasis by reagent strip test for detection of circulating cathodic antigen. J ClinMicrobiol 2004;42:5458-5461.

10. Midzi N, Butterworth AE, Mduluza T, Munyati S, Deeldere AM, van Dame GJ.Use of circulating cathodic antigen strips for the diagnosis of urinary schistosomiasis. Trans $R$ Soc Trop Med Hyg. 2009;103:45-51.

11. Nale Y, Galadima M, Yakubu SE. Seasonal changes in the distribution and infection rate of schistosome intermediate hosts in river Kubanni and its tributaries. Biosci Res Comm. 2003;15(3):2007-2011.

12. Sam-wobo SO, Ajayi TO, Ijaduola T, Ekpo UF, Adeleke MA, Surakat MA, et al. Status of urinary schistosomiasis among primary school children in Abeokuta, Nigeria. Int $\mathbf{J}$ Med. 2013;5:106-109.

13. Anosike JC, Ogwuike UT, Nwoke BEB, Asor JE, Ikpeama CA, Nwosu DC et al. Studies on vesical schistosomiasis among rural Ezza farmers in the Southwestern border of Ebonyi 
State, Nigeria. Ann Agric EnvironMed.2006; 13:13-19.

14. Ekpo UF, Laja-DeilA, OluwoleAS, Sam WoboSO Mafiana CF. Urinary schistosomiasis among preschool children in a rural community near Abeokuta, Nigeria. Parasite Vectors2010; 3:58-62.

15. Bigwan EI, Tinga B, Damen J. Prevalence of schistosomiasis among secondary school boarding students in Potiskum metropolis, Yobe State, Northern Nigeria. BAJOPAS 2012; 5:155-158.

16. Nwosu DC, Nwachukwu PC, Avoaja DA, Ajero CMU, Nwanjo HU, Obeagu GI, et al. Index of potential contamination for urinary schistosomiasis in Afikpo North L.G.A. Ebonyi State, Nigeria. Eur J Pharm Sci. 2015;2(1):439-449.

17. Akinbo, F.O., Okaka, C.E. and Omoregie, R. (2011). Proportion of urinary schistosomiasis among HIV-infected subjects in Benin City, Nigeria. Oman Med J.2011; 26(3):175-177.

18. Sanneh B, Joof E, Sanyang AM, Renneker K, Camara Y, Sey AP et al. Field evaluation of a schistosome circulating cathodic antigen rapid test kit at point-of-care for mapping of schistosomiasis endemic districts in the Gambia. PLoS One. 2017;12(8): e0182003.

\section{List of abbreviations}

CCA- Circulating Cathodic Antigen

POC- Point-of-care

NTD- Neglected Tropical Diseases

USA- United States of America

WHO- World Health Organization

CDC- Centers for Disease Control and Prevention

CSA- Circulating Schistosome Antigen

REC- Research and ethical committee

$\mathrm{MOH}-$ Ministry of Health

LGA - Local Government Area 